\title{
Adaptive Dynamic Inversion via Time-Scale Separation
}

\author{
Naira Hovakimyan, Eugene Lavretsky and Chengyu Cao
}

\begin{abstract}
This paper presents an adaptive dynamic inversion method for uncertain nonaffine-in-control systems. The adaptive dynamic inversion controller is defined as a solution of a fast dynamical equation, which achieves time-scale separation between a state predictor and the controller dynamics. Lyapunov based adaptive laws ensure that the state predictor tracks the actual nonlinear system with bounded errors. As a result, the system state tracks the reference model with bounded errors. Benefits of the proposed design method are demonstrated using Van der Pol dynamics with nonlinear control input.
\end{abstract}

\section{INTRODUCTION}

Dynamic inversion is one of the most popular methods for controlling affine-in-control minimum phase nonlinear systems [9], [11]. The main challenge in extending the methodology to nonaffine systems has to do with the fact that in the latter case the zero dynamics are not always well defined [15]. Several methods for particular classes of nonaffine systems have been reported in [10], [14], [20]. Adaptive methods for controlling nonaffine systems have been presented in [1]-[3], [5]-[7], [12].

In this paper, using the main result from [16], [19] and tools from singular perturbations theory [11], we present a dynamic inversion methodology for minimum-phase uncertain nonaffine systems with well-defined zero dynamics. To simplify the presentation, first we review the main result from [8]. The methodology in [8] invokes fast dynamics to invert the system, and hence relies on the time-scale separation property between the system dynamics and the dynamics of the inverting controller. The main idea can be illustrated by considering a scalar dynamical system of the form:

$$
\dot{x}=f(x, u), \quad x(0)=x_{0}, \quad t \geq 0,
$$

where $f$ is a known function of the system state $x$ and the control input $u$. Assuming that $\frac{\partial f}{\partial u}$ is bounded away from zero for $(x, u) \in \Omega_{x} \times \Omega_{u} \subset \mathbb{R} \times \mathbb{R}$, where $\Omega_{x}, \Omega_{u}$ are compact sets, the control objective is to find $u$ such that the state of the system (1) tracks a bounded reference input $r(t) \in \mathcal{C}^{1}$ from an arbitrary initial condition $x_{0} \in \Omega_{x}$. In [8], we prove that such a controller can be determined via the solution of the fast dynamics: $\epsilon \dot{u}=-\operatorname{sign}\left(\frac{\partial f}{\partial u}\right)(f(x, u)+$

This material is based upon work supported by the United States Air Force under Contracts No. FA9550-05-1-0157 and FA9550-04-C-0047.

Naira Hovakimyan is with Aerospace \& Ocean Engineering, Virginia Polytechnic Institute \& State University, Blacksburg, VA 24061-0203, email: nhovakim@vt.edu

Eugene Lavretsky is with Phantom Works, The Boeing Company, U.S.A. e-mail: eugene.lavretsky@boeing.com

Chengyu Cao is with Aerospace \& Ocean Engineering, Virginia Polytechnic Institute \& State University, Blacksburg, VA 24061-0203, e-mail: chengyu@vt.edu $k(x-\mathrm{r}(t))-\dot{\mathrm{r}}(t)), k>0$, where $\epsilon \ll 1$. If $\left|\frac{\partial f}{\partial u}\right|>b_{0}>0$, then for minimum phase nonlinear systems in normal form with well-defined zero dynamics, Tikhonov's theorem can be used to prove bounded tracking of a desired reference system [8].

In [13], under a set of restrictive assumptions, the method is extended to uncertain systems, and the adaptive counterpart of the method is developed. Assuming that $f$ in (1) is unknown, the methodology in [13] considers linear-inparameters neural network approximation of the unknown nonlinearity on a compact set of possible initial conditions $f(x, u)=W^{\top} \Phi(x, u)+\varepsilon(x, u)$, where $\Phi(x, u)$ is a bounded continuous regressor, $|\varepsilon(x, u)|<\varepsilon^{*}$, while $W$ is a vector of unknown constants, so that the unknown system dynamics can be presented as

$$
\dot{x}(t)=W^{\top} \Phi(x(t), u(t))+\varepsilon(x(t), u(t)) \quad x(0)=x_{0} .
$$

A state predictor using a series parallel model for the dynamics in (2) is introduced as $\dot{\hat{x}}(t)=\hat{W}^{\top}(t) \Phi(x(t), u(t))$ $a(\hat{x}(t)-x(t))$ with $a>0, \hat{x}(0)=\hat{x}_{0}$, leading to the prediction error dynamics $\dot{e}(t)=-a e(t)+\tilde{W}^{\top}(t) \Phi(x(t), u(t))-$ $\varepsilon(x(t), u(t))$, where $e(t)=\hat{x}(t)-x(t), \tilde{W}(t)=\hat{W}(t)-W$ and $e(0)=\hat{x}_{0}-x_{0}$. The projection type adaptive law

$\dot{\hat{W}}(t)=\Gamma \operatorname{Proj}(\hat{W}(t),-\Phi(x(t), u(t)) e(t)), \hat{W}(0)=W_{0}$,

where $\Gamma>0$ is the adaptation gain, ensures boundedness of the parameter errors. The control design method from [8] is applied to force the state of the predictor track the reference input $r(t)$ :

$$
\begin{aligned}
\epsilon \dot{u}(t) & =-\operatorname{sign}\left(\hat{W}^{\top}(t) \frac{\partial \Phi(x, u)}{\partial u}\right)\left(\hat{W}^{\top}(t) \Phi(x(t), u(t))\right. \\
& -a e(t)+k(\hat{x}(t)-\mathrm{r}(t))-\dot{\mathrm{r}}(t)), k>0 .
\end{aligned}
$$

Following the framework of [8], one needs to require that $\operatorname{sign}\left(\hat{W}^{\top}(t) \frac{\partial \Phi(x, u)}{\partial u}\right)$ be constant, which in its turn is crucial for exponential stability of the boundary layer system required by Tikhonov's theorem. In general, constant sign for $\hat{W}^{\top}(t) \frac{\partial \Phi(x, u)}{\partial u}$ is not easy to verify. In fact, it is not guaranteed in the most general case. In [13], we consider a special class of nonlinear systems for which a regressor vector can be introduced to ensure the constant sign for $\hat{W}^{\top}(t) \frac{\partial \Phi(x, u)}{\partial u}$. That assumption however implies that the nonaffine nature of the system dynamics is weak as compared to its affine counterpart with respect to control efficiency.

In this paper, we relax the restrictive assumption of [13] and extend the methodology to uncertain systems of more general class. We recall the main result from [16] that 
enables approximation of positive valued functions by a set of radial basis functions (RBFs) with positive coefficients. This consequently leads to consideration of a specialized set of basis functions and appropriate choice of adaptive laws such that in ensemble they verify the exponential stability property of the boundary layer system required by Tikhonov's theorem.

The paper is organized as follows. In Section II, we recall some preliminaries from approximation theory and Tikhonov's theorem from singular perturbation theory. We give our main result on tracking a given reference signal for single input systems in Section III. A relevant simulation example is given in Section IV.

\section{MAthematical Preliminaries}

\section{A. Preliminaries on Approximation Theory}

In this section, we recall the main result from [16], [19]. Let $\mathcal{C}\left(\mathbb{R}^{r}\right)$ denote the usual space of continuous maps $f$ : $\mathbb{R}^{r} \rightarrow \mathbb{R}$.

Theorem 1: [16] Let $K: \mathbb{R}^{r} \rightarrow \mathbb{R}$ be an integrable bounded function such that $K$ is continuous and $\int_{\mathbb{R}^{r}} K(x) d x \neq 0$, and $\mathcal{S}_{K}$ be the set of functions

$$
q(x)=\sum_{i=1}^{M} w_{i} K\left(\frac{x-z_{i}}{\delta}\right)
$$

where $M>0$ is a positive integer, $\delta>0, w_{i} \in \mathbb{R}$, and $z_{i} \in \mathbb{R}^{r}$. Then for any continuous function $f: \mathbb{R}^{r} \rightarrow \mathbb{R}$, for any $\varepsilon>0$ and for any compact subset $\Omega \in \mathbb{R}^{r}$ there exists a $q \in \mathcal{S}_{K}$ such that

$$
\left\|(q-f) \mathbf{1}_{\Omega}\right\|_{\infty}<\varepsilon,
$$

where $\mathbf{1}_{\Omega}$ is the indicator function of $\Omega \in \mathbb{R}^{n}$ such that

$$
\mathbf{1}_{\Omega}(x)=\left\{\begin{array}{ll}
1 & \text { if } x \in \Omega \\
0 & \text { if } x \notin \Omega
\end{array} .\right.
$$

The proof in [16] gives an explicit expression for the coefficients $w_{i}$ in (4). It shows that the coefficients are directly proportional to the corresponding function values on the compact set $\Omega$. Consequently if $f(x)>0$ for all $x \in \Omega$, then $w_{i}>0$. We also notice that the Gaussians given by $\phi\left(\frac{x-x_{c_{i}}}{\sigma_{i}}\right)=\mathrm{e}^{-\frac{\left(x-x_{c_{i}}\right)^{2}}{\sigma_{i}^{2}}}$, where $x_{c_{i}}$ is the center, while $\sigma_{i}$ is the width parameter, represent one particular choice of $K$.

\section{B. Preliminaries on Singular Perturbations Theory}

For proving our main result we will use Tikhonov's theorem on singular perturbations, which we recall below (see for instance Theorem 11.2 on page 439 of [11]).

Consider the problem of solving the system

$$
\Sigma_{0}: \begin{cases}\dot{x}(t)=f(t, x(t), u(t), \epsilon), & x(0)=\xi(\epsilon) \\ \epsilon \dot{u}(t)=g(t, x(t), u(t), \epsilon), & u(0)=\eta(\epsilon),\end{cases}
$$

where $\xi: \epsilon \mapsto \xi(\epsilon)$ and $\eta: \epsilon \mapsto \eta(\epsilon)$ are smooth. Assume that $f$ and $g$ are continuously differentiable in their arguments for $(t, x, u, \epsilon) \in[0, \infty] \times D_{x} \times D_{u} \times\left[0, \epsilon_{0}\right]$, where $D_{x} \subset \mathbb{R}^{n}$ and $D_{u} \subset \mathbb{R}^{m}$ are domains, $\epsilon_{0}>0$. In addition, let $\Sigma_{0}$ be in standard form, i.e. $0=g(t, x, u, 0)$ has $k \geq 1$ isolated real roots $u=h_{i}(t, x), \quad i \in\{1, \ldots, k\}$ for each $(t, x) \in[0, \infty] \times D_{x}$. We choose one particular $i$, which is fixed. We drop the subscript $i$ henceforth. Let $v(t, x)=u-h(t, x)$.

In singular perturbations theory, the system given by

$$
\Sigma_{00}: \quad \dot{x}(t)=f(t, x(t), h(t, x(t)), 0), \quad x(0)=\xi(0),
$$

is called the reduced system, and the system given by

$\Sigma_{b}: \frac{d v}{d \tau}=g(t, x, v+h(t, x), 0), \quad v(0)=\eta_{0}-h\left(0, \xi_{0}\right)$

is called the boundary layer system, where $\eta_{0}=\eta(0)$ and $\xi_{0}=\xi(0),(t, x) \in[0, \infty) \times D_{x}$ are treated as fixed parameters. The new time scale $\tau$ is related to the original time $t$ via the relationship $\tau=\frac{t}{\epsilon}$. The following result is due to Tikhonov [11].

Theorem 2: Consider the singular perturbation system $\Sigma_{0}$ given in (5), and let $u=h(t, x)$ be an isolated root of $g(t, x, u, 0)=0$. Assume that the following conditions are satisfied for all $[t, x, u-h(t, x), \epsilon] \in[0, \infty) \times D_{x} \times D_{v} \times$ $\left[0, \epsilon_{0}\right]$ for some domains $D_{x} \subset \mathbb{R}^{n}$ and $D_{v} \subset \mathbb{R}^{m}$, which contain their respective origins:

A1. On any compact subset of $D_{x} \times D_{v}$, the functions $f, g$, their first partial derivatives with respect to $(x, u, \epsilon)$, and the first partial derivative of $g$ with respect to $t$ are continuous and bounded, $h(t, x)$ and $\left[\frac{\partial g}{\partial u}(t, x, u, 0)\right]$ have bounded first derivatives with respect to their arguments, $\left[\frac{\partial f}{\partial x}(t, x, h(t, x))\right]$ is Lipschitz in $x$, uniformly in $t$, and the initial data given by $\xi$ and $\eta$ are smooth functions of $\epsilon$.

A2. The origin is an exponentially stable equilibrium point of the reduced system $\Sigma_{00}$ given by equation (6). There exists a Lyapunov function $V:[0, \infty) \times D_{x} \rightarrow[0, \infty)$ that satisfies

$$
\begin{gathered}
W_{1}(x) \leq V(t, x) \leq W_{2}(x) \\
\frac{\partial V}{\partial t}(t, x)+\frac{\partial V}{\partial x}(t, x) f(t, x, h(t, x), 0) \leq-W_{3}(x)
\end{gathered}
$$

for all $(t, x) \in[0, \infty) \times D_{x}$, where $W_{1}, W_{2}, W_{3}$ are continuous positive definite functions on $D_{x}$, and let $c$ be a nonnegative number such that $\left\{x \in D_{x} \mid W_{1}(x) \leq\right.$ $c$ \} is a compact subset of $D_{x}$.

A3. The origin is an equilibrium point of the boundary layer system $\Sigma_{b}$ given by equation (7), which is exponentially stable uniformly in $(t, x)$.

Let $R_{v} \subset D_{v}$ denote the region of attraction of the autonomous system $\frac{d v}{d \tau}=g\left(0, \xi_{0}, v+h\left(0, \xi_{0}\right), 0\right)$, and let $\Omega_{v}$ be a compact subset of $R_{v}$. Then for each compact set $\Omega_{x} \subset\left\{x \in D_{x} \mid W_{2}(x) \leq \rho c, 0<\rho<1\right\}$, there exists a positive constant $\epsilon_{*}$ such that for all $t \geq 0, \xi_{0} \in \Omega_{x}$, $\eta_{0}-h\left(0, \xi_{0}\right) \in \Omega_{v}$ and $0<\epsilon<\epsilon_{*}, \Sigma_{0}$ has a unique solution $x_{\epsilon}$ on $[0, \infty)$ and $x_{\epsilon}(t)-x_{00}(t)=O(\epsilon)$ holds uniformly for $t \in[0, \infty)$, where $x_{00}(t)$ denotes the solution of the reduced system $\Sigma_{00}$ in (6).

The following Remark will be useful in the sequel [11]. 
Remark 1: Assumption A3 can be locally verified by linearization. Let $\varphi$ denote the map $v \mapsto g(t, \xi, v+h(t, \xi), \epsilon)$. It can be shown that if there exists $\omega_{0}>0$ such that the Jacobian matrix $\left[\frac{\partial \varphi}{\partial v}\right]$ satisfies the eigenvalue condition $\operatorname{Re}\left(\lambda\left[\frac{\partial \varphi}{\partial v}(t, x, h(t, x), 0)\right]\right) \leq-\omega_{0}<0$ for all $(t, x) \in$ $[0, \infty) \times D_{x}$, then Assumption A3 is satisfied.

\section{TRACKING CONTROLLER}

\section{A. Problem formulation}

Consider the following nonlinear single-input system in normal form:

$$
\begin{aligned}
& \dot{x}(t)=A x(t)+B f(x(t), z(t), u(t)), x(0)=x_{0}, \\
& \dot{z}(t)=\zeta(x(t), z(t), u(t)), z(0)=z_{0},
\end{aligned}
$$

where $\left[x^{\top} z^{\top}\right]^{\top}$ denotes the state vector of the system, $x=$ $\left[\begin{array}{lll}x_{1} & \cdots & x_{r}\end{array}\right]^{\top} \in \mathbb{R}^{r}, u$ is the control input, $r$ is the relative degree of the system, $(x, z, u) \in D_{x} \times D_{z} \times D_{u}$, and $D_{x} \subset$ $\mathbb{R}^{r}, D_{z} \subset \mathbb{R}^{n-r}$ and $D_{u} \subset \mathbb{R}$ are domains containing their respective origins, $f: D_{x} \times D_{z} \times D_{u} \rightarrow \mathbb{R}, \zeta: D_{x} \times$ $D_{z} \times D_{u} \rightarrow \mathbb{R}^{n-r}$ are continuously differentiable unknown functions of their arguments, while $A$ and $B$ correspond to the controllable canonical normal form representation of the nonlinear system dynamics, i.e.

$$
A=\left[\begin{array}{cccc}
0 & 1 & \cdots & 0 \\
\vdots & \vdots & \ddots & \vdots \\
0 & 0 & \ldots & 1 \\
0 & 0 & \ldots & 0
\end{array}\right], \quad B=\left[\begin{array}{c}
0 \\
\vdots \\
0 \\
1
\end{array}\right]
$$

Assumption 1: Let $\frac{\partial f}{\partial u}$ be bounded away from zero for $(x, z, u) \in \Omega_{x, z, u} \subset D_{x} \times D_{z} \times D_{u}$, where $\Omega_{x, z, u}$ is a compact set of possible initial conditions; i.e. there exists $b_{0}>0$ such that $\left|\frac{\partial f}{\partial u}\right|>b_{0}$.

Let the reference model dynamics be given as $\dot{x}_{\mathrm{r}}(t)=$ $A_{\mathrm{r}} x_{\mathrm{r}}(t)+B_{\mathrm{r}} \mathrm{r}(t), x_{\mathrm{r}}(0)=x_{\mathrm{r}, 0}$, where $\mathrm{r}(t)$ is a continuous bounded reference input signal, $x_{r}=\left[\begin{array}{lll}x_{r, 1} & \cdots & x_{r, r}\end{array}\right]^{\top} \in \mathbb{R}^{r}$ is the state of the reference model, and the Hurwitz matrix $A_{\mathrm{r}}$ and the column vector $B_{\mathrm{r}}$ have the following structure:

$$
A_{\mathrm{r}}=\left[\begin{array}{cccc}
0 & 1 & & \\
\vdots & & \ddots & \\
0 & & & 1 \\
-a_{1} & -a_{2} & \ldots & -a_{r}
\end{array}\right], \quad B_{\mathrm{r}}=\left[\begin{array}{c}
0 \\
\vdots \\
0 \\
b
\end{array}\right] .
$$

The control objective is to design a tracking control law to ensure that $x(t) \rightarrow x_{\mathrm{r}}(t)$ as $t \rightarrow \infty$, while all other error signals remain bounded.

\section{B. RBF approximation}

Let $g(x, z, u)=\frac{\partial f}{\partial u}(x, z, u)$. Following Assumption 1, $|g(x, z, u)|>b_{0}>0$ for all $(x, z, u) \in \Omega_{x, z, u} \subset D_{x} \times D_{z} \times$ $D_{u}$. Without loss of generality, let $g(x, z, u)>b_{0}>0$. Let $\mathcal{F}$ denote the family of all continuous functions $f(x, z, u)$ in $\Omega_{x, z, u}$ monotonic with respect to $u$, i.e. for all $f \in \mathcal{F}$ we have $g(x, z, u)>b_{0}>0$.
For all possible $M, N, \theta_{i} \in \mathbb{R}$ and all possible positive $w_{i} \in \mathbb{R}^{+}$consider the following family of functions $\phi$ : $\Omega_{x, z, u} \rightarrow \mathbb{R}:$

$$
\phi(x, z, u)=\phi_{1}(x, z)+\phi_{2}(x, z, u),
$$

where

$$
\begin{aligned}
\phi_{1}(x, z) & \triangleq \theta^{\top} \Phi_{1}(x, z)=\sum_{i=1}^{N} \theta_{i} \mathrm{e}^{-\frac{\left(y-y_{c_{i}}\right)^{\top}\left(y-y_{c_{i}}\right)}{\delta_{i}^{2}}}(10) \\
y & \triangleq\left[x^{\top} z^{\top}\right]^{\top}, \delta_{i}>0, \\
\phi_{2}(x, z, u) & \triangleq w^{\top} \Phi_{2}(x, z, u) \\
& =\sum_{j=1}^{M} w_{j}\left(\int_{0}^{u} \mathrm{e}^{-\frac{\left(\chi-\chi c_{j}\right)^{\top}\left(\chi-\chi c_{j}\right)}{\sigma_{j}^{2}}} d \xi\right), \\
\chi & \triangleq\left[x^{\top} z^{\top} \xi\right]^{\top}, \sigma_{j}>0,
\end{aligned}
$$

where $\xi$ is introduced to denote the integration variable, $\Phi_{1}(x, z)$ is the vector of Gaussians independent of $u$, while $\Phi_{2}(x, z, u)$ is the vector of the integrals of the Gaussians dependent upon $u$. The vectors $y_{c_{i}} \triangleq\left[\begin{array}{ll}x_{c_{i}}^{\top} & z_{c_{i}}^{\top}\end{array}\right]^{\top}, i=$ $1, \cdots, N, \chi_{c_{j}}=\left[\begin{array}{lll}x_{c_{j}}^{\top} & z_{c_{j}}^{\top} & u_{c_{j}}\end{array}\right]^{\top}, j=1, \cdots, M$ represent the fixed centers of the basis, $\delta_{i}, \sigma_{j}$ are the fixed width parameters, while $\theta_{i} \in \mathbb{R}, w_{j} \in \mathbb{R}^{+}$are the unknown constant parameters. We denote this family of functions by $\mathcal{S}$. It is straightforward to verify from (9)-(11) that for all $\phi(x, z, u) \in \mathcal{S}$

$$
\operatorname{sgn}\left(\frac{\partial \phi}{\partial u}(x, z, u)\right)=\operatorname{sgn}\left(\frac{\partial \phi_{2}}{\partial u}(x, z, u)\right)>0 .
$$

We note that $\mathcal{F} \subset \mathcal{C}\left(\mathbb{R}^{n+1}\right)$ and $\mathcal{S} \subset \mathcal{C}\left(\mathbb{R}^{n+1}\right)$. A straightforward corollary from Theorem 1 is given by the following proposition.

Proposition 1: $\mathcal{S}$ is dense in $\mathcal{F}$ uniformly with respect to $\|\cdot\|_{\infty}$ norm.

Proof. First we notice that for any $f(x, z, u)$ from the class $\mathcal{F}$ the following representation is true:

$$
f(x, z, u)=f(x, z, 0)+\int_{\xi=0}^{u} g(x, z, \xi) d \xi .
$$

Since $f(x, z, 0) \in \mathcal{C}\left(\mathbb{R}^{n+1}\right)$, it follows from Theorem 1 that $f(x, z, 0)$ can be approximated arbitrarily closely by a $\phi_{1}$ type function from (10) over $(x, z) \in \Omega_{x, z}$, i.e. $\left\|f(x, z, 0)-\phi_{1}(x, z)\right\|_{\infty} \leq \varepsilon_{1}$, where $\varepsilon_{1}>0$. Since $g(x, z, u) \in \mathcal{C}_{+}\left(\mathbb{R}^{n+1}\right)$, it follows from Theorem 1 that for arbitrary $\varepsilon_{2}>0$ there exists positive valued $q^{+} \in \mathcal{S}_{G}$ such that for $(x, z, u) \in \Omega_{x, z, u} \subset D_{x} \times D_{z} \times D_{u}$ one has $\left\|g(x, z, u)-q^{+}(x, z, u)\right\|_{\infty} \leq \varepsilon_{2}$, which leads to the following upper bound

$$
\begin{aligned}
& \left\|\int_{\xi=0}^{u}\left(g(x, z, \xi)-q^{+}(x, z, \xi)\right) d \xi\right\|_{\infty} \\
& \leq \int_{\xi=0}^{u}\left\|g(x, z, \xi)-q^{+}(x, z, \xi)\right\|_{\infty} d \xi \\
& \leq \int_{\xi=0}^{u} \varepsilon_{2} d \xi \leq \varepsilon_{2} \int_{\xi=0}^{u} d \xi=\varepsilon_{2} u .
\end{aligned}
$$


Since the approximation is considered over the compact set $(x, z, u) \in \Omega_{x, z, u}$, then there exist finite $\rho>0$ such that $|u| \leq \rho$. Hence,

$$
\left\|\int_{\xi=0}^{u} g(x, z, \xi) d \xi-\int_{\xi=0}^{u} q^{+}(x, z, \xi) d \xi\right\|_{\infty} \leq \rho \varepsilon_{2}
$$

for any arbitrary small $\varepsilon_{2}$. This implies that the second term in (13) can be approximated arbitrarily closely by a $\phi_{2}$ type function from (11). Hence, for arbitrary $\varepsilon^{*}>0$ there exists $\phi(x, z, u) \in \mathcal{S}$ such that $\|f(x, z, u)-\phi(x, z, u)\|_{\infty} \leq \varepsilon^{*}$ for all $(x, z, u) \in \Omega_{x, z, u} \subset D_{x} \times D_{z} \times D_{u}$. The proof is complete.

Following the statement in Proposition 1, consider the following approximation for the unknown function $f(x, z, u)$ in system dynamics (8) via the family of functions $\phi$ : $\Omega_{x, z, u} \rightarrow \mathbb{R}$ so that $f(x, z, u)=W^{\top} \Phi(x, z, u)+\varepsilon(x, z, u)$, where $(x, z, u) \in \Omega_{x, z, u},|\varepsilon(x, z, u)| \leq \varepsilon^{*}, W=\left[\begin{array}{ll}\theta^{\top} & w^{\top}\end{array}\right]^{\top}$, $\Phi(x, z, u)=\left[\begin{array}{ll}\Phi_{1}^{\top}(x, z) & \Phi_{2}^{\top}(x, z, u)\end{array}\right]^{\top}$, and $w_{i}$ 's are positive.

\section{State predictor}

Consider the following one-step-ahead state predictor using a series parallel model for the dynamics in (8):

$\dot{\hat{x}}(t)=A \hat{x}(t)+B\left(\hat{W}^{\top}(t) \Phi(x(t), z(t), u(t))-\sum_{i=1}^{i=r} a_{s, i} e_{s, i}(t)\right)$,

with $\hat{x}(0)=x_{0}$, where $e_{s}(t)=\hat{x}(t)-x(t)$ is the prediction error signal, $a_{s, i}>0$ define a Hurwitz polynomial, while $\hat{W}(t)$ is an adaptive parameter for estimating the unknown constant vector $W$. Then the prediction error dynamics for the series parallel model in (16) are:

$$
\begin{aligned}
\dot{e}_{s}(t) & =A_{s} e_{s}(t)+B\left(\tilde{W}^{\top}(t) \Phi(x(t), z(t), u(t))\right. \\
& -\varepsilon(x(t), z(t), u(t))) \\
\dot{z}(t) & =\zeta(x(t), z(t), u(t))
\end{aligned}
$$

with $e_{s}(0)=0, z(0)=z_{0}, \tilde{W}(t)=\hat{W}(t)-W$, where $A_{s}$ has the same structure of $A_{\mathrm{r}}$ except for the last row being comprised of the coefficients $a_{s, i}$ instead of $a_{i}$. The proof of the next lemma follows from the properties of the projection operator [17].

Lemma 1: The adaptive law

$$
\dot{\hat{W}}(t)=\Gamma \operatorname{Proj}\left(\hat{W}(t),-\Phi(x(t), z(t), u(t)) e_{s}^{\top}(t) P B\right),
$$

where $\operatorname{Proj}(\cdot, \cdot)$ denotes the Projection operator [17], $P=$ $P^{\top}>0$ solves the Lyapunov equation $A_{s}^{\top} P+P A_{s}=$ $-Q$ for arbitrary $Q>0, \Gamma>0$ is the adaptation gain matrix, ensures that the parametric errors $\tilde{W}(t)$ are ultimately bounded.

Remark 2: Notice that since $w_{i}$ are positive, the compact set in the application of the Projection operator can be selected in a way so that $\hat{w}_{i}(t)$ remain positive for all $t \geq 0$, i.e. $\hat{w}_{i}(t)>w_{i_{0}}>0$ for all $i=1, \cdots, M$.

Remark 3: Notice that ultimate boundedness of parametric errors, stated in Theorem 1, does not imply stability of the overall system. One needs to construct a bounded $u(t)$ and prove in addition that in the presence of this feedback one of the systems, (8) or (16), remains bounded and achieves the tracking objective. We will apply the methodology from [8] to force the state of the predictor in (16) track the desired reference input. Boundedness of the system state will follow.

\section{Control design}

Let $e(t)=\hat{x}(t)-x_{\mathrm{r}}(t)$ be the tracking error signal between the series parallel model and the reference system. Then the open loop (time-varying) tracking error dynamics are given by:

$$
\begin{aligned}
\dot{e}(t) & =F\left(\hat{x}(t), x(t), e_{s}(t), z(t), u(t), \hat{W}(t)\right) \\
& -A_{\mathrm{r}} x_{\mathrm{r}}(t)-B_{\mathrm{r}} \mathrm{r}(t), \quad e(0)=e_{0} \\
\dot{z}(t) & =\zeta(x(t), z(t), u(t)), \quad z(0)=z_{0},
\end{aligned}
$$

where $F\left(\hat{x}, x, e_{s}, z, u, \hat{W}\right)=\left[\hat{x}_{2}, \cdots, \hat{x}_{r}, \hat{W}^{\top} \Phi(x, z, u)-\right.$ $\left.\sum_{i=1}^{i=r} a_{s, i} e_{s, i}\right]^{\top}$. Dynamic inversion based controller is defined for the state predictor as the solution of

$$
\hat{W}^{\top} \Phi(x, z, u)-\sum_{i=1}^{i=r} a_{s, i} e_{s, i}=-\sum_{i=1}^{i=r} a_{i} \hat{x}_{i}+b r
$$

resulting in the asymptotically stable closed-loop tracking error dynamics $\dot{e}(t)=A_{\mathrm{r}} e(t)$. Since (22) cannot (in general) be solved explicitly for $u$, we construct an approximation of the dynamic inversion controller by introducing the following fast dynamics:

$$
\epsilon \dot{u}=-\operatorname{sign}\left(\frac{\partial \mathbf{f}}{\partial u}\right) \mathbf{f}(t, e, z, u), \quad u(0)=u_{0},
$$

where

$$
\begin{aligned}
& \mathbf{f}(t, e, z, u)=\hat{W}^{\top}(t) \Phi\left(e+x_{\mathrm{r}}(t)-e_{s}(t), z, u\right) \\
& -\sum_{i=1}^{i=r} a_{s, i} e_{s, i}(t)+\sum_{i=1}^{i=r} a_{i}\left(e_{i}+x_{\mathrm{r}, i}(t)\right)-b \mathrm{r}(t) .
\end{aligned}
$$

Let $u=h(t, e, z)$ be an isolated root of $\mathbf{f}(t, e, z, u)=0$. The reduced system for (20)-(21) is given by:

$$
\begin{gathered}
\dot{e}(t)=A_{\mathrm{r}} e(t), \quad e(0)=e_{0} \\
\dot{z}(t)=\zeta\left(x_{\mathrm{r}}(t)+e(t)-e_{s}(t), z(t), h(t, e(t), z(t))\right.
\end{gathered}
$$

with $z(0)=z_{0}$. The boundary layer system is:

$$
\frac{d v}{d \tau}=-\operatorname{sign}\left(\frac{\partial \mathbf{f}}{\partial u}\right) \mathbf{f}(t, e, z, v+h(t, e, z)) .
$$

Tikhonov's theorem leads to the following result.

Theorem 3: Assume that the following conditions are satisfied for all $[t, e, z, u-h(t, e, z), \epsilon] \in[0, \infty) \times D_{e, z} \times$ $D_{v} \times\left[0, \epsilon_{0}\right]$ for some domains $D_{e, z} \subset \mathbb{R}^{n}$ and $D_{v} \subset \mathbb{R}$, which contain their respective origins:

B1. On any compact subset of $D_{e, z} \times D_{v}$, the functions $\mathbf{f}, \zeta$, and their first partial derivatives with respect to $(e, z, u)$, and the first partial derivative of $\mathbf{f}$ with respect to $t$ are continuous and bounded, $h(t, e, z)$ and $\frac{\partial \mathbf{f}}{\partial u}(t, e, z, u)$ have bounded first derivatives with respect to their arguments, $\frac{\partial \mathbf{f}}{\partial e}, \frac{\partial \mathbf{f}}{\partial z}$ as functions of $(t, e, z, h(t, e, z))$ are Lipschitz in $e, z$, uniformly in $t$. 
B2. The origin is an exponentially stable equilibrium point of the system $\dot{z}(t)=\zeta\left(x_{r}(t)-e_{s}(t), z(t), h(t, 0, z(t))\right.$. The map $(e, z) \mapsto \zeta\left(e+x_{\mathrm{r}}(t)-e_{s}(t), z, h(t, e, z)\right)$ is continuously differentiable and Lipschitz in $(e, z)$, uniformly in $t$.

B3. $(t, e, z, v) \mapsto \frac{\partial f}{\partial u}(t, e, z, v+h(t, e, z))$ is bounded below by some positive number for all $(t, e, z) \in[0, \infty) \times$ $D_{e, z}$.

Then the origin of (27) is exponentially stable. Moreover, let $\Omega_{v}$ be a compact subset of $R_{v}$, where $R_{v} \subset D_{v}$ denotes the region of attraction of the autonomous system $\frac{d v}{d \tau}=-\operatorname{sign}\left(\frac{\partial \mathbf{f}}{\partial u}\right) \mathbf{f}\left(0, e_{0}, z_{0}, v+h\left(0, e_{0}, z_{0}\right)\right)$. Then for each compact subset $\Omega_{z, e} \subset D_{z, e}$ there exists a positive constant $\epsilon_{*}$ and a $T>0$ such that for all $t \geq 0,\left(e_{0}, z_{0}\right) \in \Omega_{e, z}$, $u_{0}-h\left(0, e_{0}, z_{0}\right) \in \Omega_{v}$ and $0<\epsilon<\epsilon_{*}$, the system of equations (16), (23) has a unique solution $\hat{x}_{\epsilon}(t)$ on $[0, \infty)$, and $\hat{x}_{\epsilon}(t)=x_{\mathrm{r}}(t)+O(\epsilon)$ holds uniformly for $t \in[T, \infty)$.

Proof. We need to verify that Assumptions A1, A2, A3 in Tikhonov's Theorem are satisfied. Assumption B1 clearly implies that A1 holds.

We now show that Assumption A2 holds. Assumption B2 implies (see Lemma 4.6, page 176 of [11]), that the system $\dot{z}(t)=\zeta\left(x_{r}(t)-e_{s}(t)+e(t), z(t), h\left(t, x_{r}(t)-e_{s}(t)+\right.\right.$ $e(t), z(t)$ ) (with $e$ viewed as the input) is input to state stable. Thus, there exist class $\mathcal{K}$ and class $\mathcal{K} \mathcal{L}$ functions $\gamma$ and $\beta$, respectively, such that $\|z(t)\| \leq \beta\left(\left\|z\left(t_{0}\right)\right\|, t-\right.$ $\left.t_{0}\right)+\gamma\left(\sup _{t_{0} \leq \tau \leq t}\|e(\tau)\|\right)$ for all $t \geq t_{0}, t_{0} \in[0, \infty)$. Furthermore, from the proof of Lemma 4.6 of [11], it follows that $\gamma(\rho)=c \rho$, for some constant $c>0$. Using the fact that the unforced system $\dot{z}=\zeta\left(x_{\mathrm{r}}-e_{s}, z, h(t, 0, z)\right)$ has 0 as an exponentially stable equilibrium point, it can be seen from the proof of Lemma 4.6 of [11] that $\beta(\rho, t)=$ $k \rho \mathrm{e}^{-\omega t}$ for some positive constants $k$ and $\omega$. Thus the solution to the reduced system (25)-(26) satisfies $\|e(t)\| \leq$ $\left\|e_{0}\right\| c_{1} \mathrm{e}^{-\omega_{0} t}$ and $\|z(t)\| \leq\left(\left\|x_{0}\right\|+\left\|z_{0}\right\|\right) c_{2} \mathrm{e}-\omega_{0} t$ for all $t \geq 0$ and for some $\omega_{0}>0$. Hence, the origin $(0,0)$ is an exponentially stable equilibrium point of (25)-(26). From a converse Lyapunov theorem it follows that there exists a Lyapunov function $V:[0, \infty) \times D_{e, z} \rightarrow \mathbb{R}$ such that $w_{1}\|(e, z)\|^{2} \leq V(t, e, z) \leq w_{2}\|(e, z)\|^{2}$ and $\frac{\partial V}{\partial t}(t, e, z)+$ $\nabla_{e, z} V \cdot \mathbf{F}(t, e, z) \leq-w_{3}\|(e, z)\|^{2}$, where $\mathbf{F}(t, e, z)=$ $\left[\left(A_{\mathrm{r}} e\right)^{\top} \zeta^{\top}\left(e+x_{\mathrm{r}}(t)-e_{s}(t), z, h(t, e, z)\right)\right]^{\top}$, [11]. We note that any positive $c$ can be chosen in A2 of Tikhonov's Theorem, and so $\Omega_{e, z} \subset\left\{(e, z) \in D_{e, z} \mid W_{2}(e, z) \leq\right.$ $\rho c, 0<\rho<1\}$ can be any compact subset of $D_{e, z}$.

In the light of Remark 1 it is easy to see that with the definition of the boundary layer system, given by (27), its exponential stability can be verified locally by linearization with respect to $v$. Indeed, since $\Phi_{1}(x, z)$ is independent of $u$, it follows from (24) that

$$
\frac{\partial \mathbf{f}(t, e, z, u)}{\partial u}=\hat{w}^{\top}(t) \frac{\partial \Phi_{2}(t, e, z, u)}{\partial u} .
$$

As stated in Remark 3, since $w_{i}$ are positive, the compact set in the application of the Projection operator can be selected such to ensure that $\hat{w}_{i}(t)>0$. Therefore, using the condition in (12) we conclude that

$$
\operatorname{sgn}\left(\frac{\partial \mathbf{f}(t, e, z, u)}{\partial u}\right)=\operatorname{sgn}\left(\frac{\partial \Phi_{2}(t, e, z, u)}{\partial u}\right)>0 .
$$

Thus, the linearization of (27) around its origin implies that the boundary layer system has locally exponentially stable origin. Hence, Tikhonov's theorem applies and so it follows that for each compact set $\Omega_{e, z} \subset D_{e, z}$ there exists a positive constant $\epsilon_{*}$ and such that for all $\left(e_{0}, z_{0}\right) \in \Omega_{e, z}$, $u_{0}-h\left(0, e_{0}, z_{0}\right) \in \Omega_{v}$ and $0<\epsilon<\epsilon_{*}$, the system of equations given by (16), (23) has a unique solution $\hat{x}_{\epsilon}, z_{\epsilon}$ on $[0, \infty)$, and $\hat{x}_{\epsilon}(t)=x_{\mathrm{r}}(t)+O(\epsilon), z_{\epsilon}(t)=z_{\mathrm{r}}(t)+O(\epsilon)$ hold uniformly for $t \in[T, \infty), z_{\mathrm{r}}$ being the solution of

$$
\begin{aligned}
& \dot{e}(t)=A_{\mathrm{r}} e(t) \\
& \dot{z}(t)=\zeta\left(x_{\mathrm{r}}(t)-e_{s}(t)+e(t), z(t), h(t, e(t), z(t))\right)
\end{aligned}
$$

with $e(0)=e_{0}, z(0)=z_{0}$, and $T \geq 0$ is such that $\| \mathrm{e}^{T A_{\mathrm{r}}} x_{0}-$ $\mathrm{e}^{T A_{\mathrm{r}}} x_{\mathrm{r}, 0} \| \leq \epsilon$.

Corollary 1: From Lemma 1 and Theorem 3 it follows that $x(t)$ tracks $x_{\mathrm{r}}(t)$ with bounded errors.

Proof. Indeed, application of Tikhonov's Theorem implies that:

$$
\hat{x}_{\epsilon}(t)=x_{\mathrm{r}}(t)+O(\epsilon)
$$

holds uniformly for $t \in[T, \infty)$. Thus, there exists a compact set $\Omega_{\hat{x}}$ such that $\hat{x}(t) \in \Omega_{\hat{x}}$ for all $t \geq 0$. Choosing $\Omega_{x, z, u}=\Omega_{\hat{x}} \times \Omega_{z} \times \Omega_{u}$ be the set of RBF distribution, standard Lyapunov arguments can be applied to prove that the projection based adaptation law in (19) ensures that the prediction error $e_{s}(t)$ remains ultimately bounded. Recalling that $x_{r}(t)=\hat{x}(t)-e(t)=x(t)+e_{s}(t)-e(t)$, we get that as $t \rightarrow \infty$ the tracking error $x(t)-x_{r}(t)$ is ultimately bounded.

\section{Simulations}

Consider the following Van der Pol oscillator driven by nonlinear control input:

$$
\begin{aligned}
\dot{x} & =A x+B\left(-x_{1}+\left(1-x_{1}^{2}\right) x_{2}+\tanh \left(x_{1}+u+3\right)\right. \\
& +\tanh (u-3)+0.01 u)
\end{aligned}
$$

where $A=\left[\begin{array}{ll}0 & 1 \\ 0 & 0\end{array}\right], B=\left[\begin{array}{l}0 \\ 1\end{array}\right]$, and $x=\left[\begin{array}{ll}x_{1} & x_{2}\end{array}\right]^{\top} \in$ $\mathbb{R}^{2}$ is the state vector available as measurement, $u \in \mathbb{R}$ is the control signal, and $x(0)=\left[\begin{array}{ll}0 & 0\end{array}\right]^{\top}$. It is easy to see that the system dynamics are invertible, but not in terms of elementary functions. The linear component $0.01 u$ is added to keep the control efficiency bounded away from zero in the entire space of variables. Simulation is performed using the following reference parameters $A_{\mathrm{r}}=\left[\begin{array}{cc}0 & 1 \\ -2 & -3\end{array}\right], B_{\mathrm{r}}=\left[\begin{array}{l}0 \\ 2\end{array}\right]$, and $\mathrm{r}(t)=$ $\frac{1}{1+\mathrm{e}^{t-8}}-\frac{1}{1+\mathrm{e}^{t-15}}+\frac{0.25}{1+\mathrm{e}^{t-30}}+0.5$ with zero initial conditions. The approximation of the nonlinearity is done with the use of 150 RBFs. Among them, 25 are $\Phi_{1}(x)$-type Gaussians distributed over the grid $x_{1} \in[-2,2], x_{2} \in[-2,2]$ with the step size equal to 1 in both dimensions and the width $\delta=1$. We use $125 \Phi_{2}(x, u)$-type basis functions, distributed 
over the grid $x_{1} \in[-2,2], x_{2} \in[-2,2]$, with step size equal to 1 , and $u \in[-4,4]$ with step size equal to 2 and the width set to $\sigma=2$ in all dimensions. The norm upper bound for the projection operator is selected as $W^{*}=10$, the lower bound for the positive weights $w$ is set to 0.01 , and the adaptation gain is $\Gamma=0.2$. We use the following state predictor with zero initialization:

$$
\begin{gathered}
\dot{\hat{x}}(t)=A \hat{x}(t)+B\left(\hat{\theta}^{\top}(t) \Phi_{1}(x(t))+\hat{w}^{\top}(t) \Phi_{2}(x(t), u(t))\right) \\
-\left[\begin{array}{cr}
0 & 0 \\
25 & 10
\end{array}\right](\hat{x}(t)-x(t)) .
\end{gathered}
$$

Denoting by $e(t)=\hat{x}(t)-x_{\mathrm{r}}(t)$ the error between the state predictor and the reference system, we have $\dot{e}(t)=A_{\mathrm{r}} e(t)-$ $B_{\mathrm{r}} \mathrm{r}(t)+\left[\begin{array}{ll}0 & \kappa(t)\end{array}\right]^{\top}$, where $\kappa(t)=\hat{\theta}^{\top}(t) \Phi_{1}(x(t))+$ $\hat{w}^{\top}(t) \Phi_{2}(x(t), u(t))-\left[\begin{array}{ll}25 & 10\end{array}\right](\hat{x}(t)-x(t))+\left[\begin{array}{ll}2 & 3\end{array}\right]^{\top} \hat{x}(t)$. The control signal needs to solve $\kappa=2 \mathrm{r}$. The fast dynamics

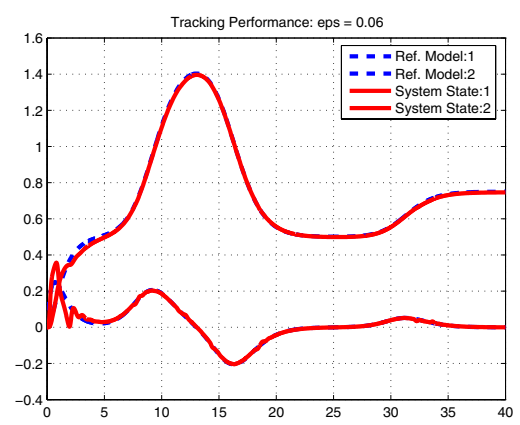

Fig. 1. Tracking performance

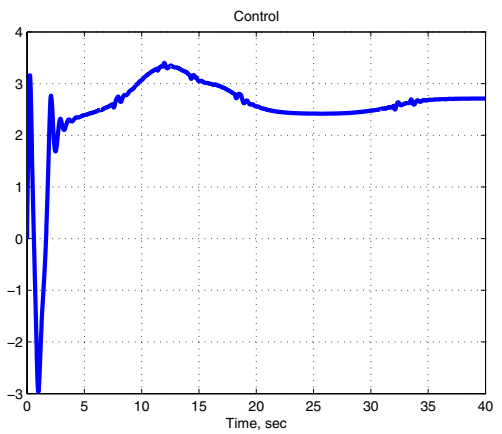

Fig. 2. Control signal

for determining the solution of it are designed as:

$$
\begin{gathered}
0.02 \dot{u}(t)=2 r(t)+\left[\begin{array}{ll}
25 & 10
\end{array}\right](\hat{x}(t)-x(t))-\left[\begin{array}{ll}
2 & 3
\end{array}\right]^{\top} \hat{x}(t) \\
-\hat{\theta}^{\top}(t) \Phi_{1}(x(t))-\hat{w}^{\top}(t) \Phi_{2}(x(t), u(t)), u(0)=0 .
\end{gathered}
$$

Figure 1 shows the closed-loop tracking performance of the reference reference state $x_{r}(t)$ by actual system state $x(t)$, while Figure 2 shows the actual control effort $u(t)$. We note that all the states and control variable remain within the domain of RBF approximation.

\section{Conclusions}

In this paper, we presented a new design technique for adaptive dynamic inversion of nonaffine-in-control uncertain systems. Using the main result from [16], we proposed a new family of basis functions that respects the monotonic property with respect to control input of the unknown system dynamics. Using this class of approximators, we designed a state predictor and used tools from singular perturbation theory to achieve the desired tracking objective for the state predictor. With the projection type of adaptive law, standard Lyapunov arguments implied that the state predictor tracks the system state with bounded errors. As a result, the proposed control enables the system state to track the reference model with bounded errors.

\section{REFERENCES}

[1] L. Chen, J. Boskovic and R. Mehra. Adaptive control design for nonaffine models arising in flight control. AIAA Journal of Guidance, Control, and Dynamics, 27(2):209-217, 2004.

[2] A. J. Calise and R.T. Rysdyk. Nonlinear adaptive flight control using neural networks. IEEE Control System Magazine, 18(6):14-25, 1998.

[3] A. J. Calise, N. Hovakimyan and M. Idan. Adaptive output feedback control of nonlinear systems using neural networks. Automatica, 37(8):1201-1211, 2001.

[4] O. Egeland, A.S. Shiriaev, H. Ludvigsen and A.L. Fradkov. Swinging up of non-affine in control pendulum. In Proc. of American Control Conference, pp. 4039-4044, 1999.

[5] S.S. Ge and J. Zhang. Neural-network control of nonaffine nonlinear system with zero dynamics by state and output feedback. IEEE Trans. Neural Networks, 14(4):900-918, 2003.

[6] N. Hovakimyan, F. Nardi and A. Calise. A novel error observer based adaptive output feedback approach for control of uncertain systems. IEEE Trans. Autom. Contr., 47(8):1310-1314, 2002.

[7] N. Hovakimyan, F. Nardi, A. Calise. and N. Kim. Adaptive Output Feedback Control of Uncertain Systems using Single Hidden Layer Neural Networks. IEEE Trans. Neural Networks, 13(6), pp.1420-1431, 2002.

[8] N. Hovakimyan, E. Lavretsky and A. Sasane. Dynamic inversion for nonaffine-in-control systems via time-scale separation: Part I. In Proc. of American Control Conference, pp. 3542-3547, 2005.

[9] A. Isidori. Nonlinear Control Systems. Springer, 1995.

[10] D. Fontaine and P. Kokotovic. Approaches to global stabilization of a nonlinear system not affine in control. In Proc. of American Control Conference, pp. 1645-1647, 1998.

[11] H.K. Khalil. Nonlinear Systems. Prentice Hall, 2002.

[12] P. Krishnamurthy and F. Khorrami. A high-gain scaling technique for adaptive output feedback control of feedforward systems. IEEE Trans. on Autom. Contr., 49(12):2286- 2292, 2004.

[13] E. Lavretsky and N. Hovakimyan. Adaptive dynamic inversion for nonaffine-in-control systems via time-Scale separation: Part II. In Proc. of American Control Conference, pp. 3548-3553, 2005.

[14] E. Moulay and W. Perruquetti. Stabilization of nonaffine systems: a constructive method for polynomial systems. IEEE Trans. Autom. Contr, 50(4):520-526, 2005.

[15] D. Nesic, E. Skafidas, I.M.Y. Mareels and R.J. Evans, Minimum phase properties for input nonaffine nonlinear systems. IEEE Trans. on Autom. Contr., 44(4):868 - 872, 1999.

[16] J. Park and I.W. Sandberg. Universal Approximation using RadialBasis-Function Networks. Neural Computation, No.3: 246-257, 1991

[17] J.B. Pomet and L. Praly. Adaptive nonlinear regulation: estimation from the Lyapunov equation. IEEE Trans. Autom. Contr., 37(6):729740, 1992.

[18] W. Rudin. Real and Complex Analysis. McGraw Hill, NY, 1986.

[19] F. Scarselli and A. C. Tsoi. Universal approximation using feedforward neural networks: A survey of some existing methods, and some new results. Neural Networks, 11(1):15-37, 1998.

[20] R. Sepulchre, M. Jankovic and P. Kokotovic. Constructive Nonlinear Control. Springer, 1997. 